EPV181/\#221 CARBOPLATIN-BASED HYPERTHERMIC INTRAPERITONEAL CHEMOTHERAPY FOR MANAGING ADVANCED PRIMARY AND RECURRENT OVARIAN CARCINOMA - A SINGLECENTER EXPERIENCE

${ }^{1} \mathrm{C}$ Brault, ${ }^{2} \mathrm{~A}$ Brind'Amour, ${ }^{3} \mathrm{~L}$ De Guerké, ${ }^{3} \mathrm{M}-\mathrm{H}$ Auclair, ${ }^{2} \mathrm{~L}$ Sideris, ${ }^{2} \mathrm{P}$ Dubé, ${ }^{3} \mathrm{~S}$ Fortin. ${ }^{1}$ University of Montreal, Obstetrics and Gynecology, Montreal, Canada; ${ }^{2}$ Hôpital Maisonneuve-Rosemont, Surgical Oncology, Montreal, Canada; ${ }^{3}$ Hôpital MaisonneuveRosemont, Gynecologic Oncology, Montreal, Canada

10.1136/ijgc-2021-IGCS.252

Objectives Studies have highlighted the benefits of combining cytoreductive surgery with HIPEC to improve survival in primary and recurrent EOC. However, data regarding the use of carboplatin-based HIPEC is limited, but seems promising in a few studies. It has lower rates of adverse effects, especially nephrotoxicity, with systemic use compared to cisplatin. In efforts to minimize morbidity, carboplatin is an important alternative to consider compared to standard HIPEC regimen.

Methods We retrospectively evaluated patients with advanced EOC who underwent CRS combined with carboplatin-based HIPEC at our center since 2013. Data collected included patients' demographics, surgical morbidity and outcomes.

Results We identified 54 patients with a median age of 60 years. There were 48 patients with primary disease and 6 with recurrent EOC. The median peritoneal cancer index was 13 and complete cytoreduction was achieved in 49 patients (91\%). Median hospital stay was 14 days and there were 6 admissions to ICU (11\%) and 7 readmissions (13\%). Severe adverse events occurred in 12 patients (22\%) and there was no perioperative or postoperative death. Recurrence was seen in 37 patients $(73 \%)$ with a median disease-free survival of 13.0 months and overall survival of 26.0 months. Cox multivariate analyses showed that completeness of cytoreduction had a significant impact on DFS. Age, PCI, occurrence of severe complications, and bowel resection did not significantly alter DFS and OS in our cohort.

Conclusions Extensive CRS combined with carboplatin-based HIPEC for advanced EOC presents acceptable morbidity and outcomes in our cohort. Larger studies are required to determine long-term oncological outcomes.

\section{EPV182/\#225 LAPAROSCOPIC INTERVAL DEBULKING SURGERY FOR ADVANCED OVARIAN CANCER-SINGLE CENTRE EXPERIENCE}

${ }^{1} Y$ Naaman*, ${ }^{1} D$ Neesham, ${ }^{1} \mathrm{~A}$ Jones, ${ }^{1} \mathrm{R}$ Mcbain, ${ }^{1} \mathrm{E}$ Vicario, ${ }^{2} \mathrm{O}$ Mcnally. ${ }^{1}$ The Royal Women's Hospital, Melbourne, Gynaecology-oncology, Parkville, Australia; ${ }^{2}$ Royal Women's Hospital, Gynaeoncology Unit, Parkville, Australia

\subsection{6/ijgc-2021-IGCS.253}

Objectives To assess the feasibility and safety of laparoscopic interval debulking surgery for patients with advanced ovarian cancer.

Methods Retrospective case series of laparoscopic interval debulking surgery for selected patients with advanced ovarian cancer (stages III/IV) between October 2017 and October 2020 in our unit.
Results In our series of sixty patients, an Optimal debulking $(\mathrm{R}<1 \mathrm{~cm})$ was achieved in 55 cases $(92 \%)$. Conversion to Midline Laparotomy was performed in 2 cases $(3 \%)$. The mean length of stay was 3.33 days (2-13 days). While the overall complication rate was low, there were 3 cases $(5 \%)$ of inadvertent transverse colon mesentery injury recognised at the time of omentectomy that necessitated bowel resection. Short term follow-up prognostic outcome is comparable to the reported outcome for laparotomy IDB cases.

Conclusions Laparoscopic IDB in selected cases is feasible and effective in achieving optimal debulking. However, surgeons should be aware of specific possible complications related to this procedure.

\section{EPV183/\#227 PARP INHIBITORS IN NEWLY DIAGNOSED ADVANCED OVARIAN CANCER: AN ASSESSMENT OF CLINICAL PRACTICES}

${ }^{1} \mathrm{G}$ Fisher ${ }^{*},{ }^{2} \mathrm{~A}$ Furedy, ${ }^{2} \mathrm{~J}$ Vandenbroucque, ${ }^{3} \mathrm{~J}$ Ledermann. ${ }^{1}$ Medscape, Clinical Strategy, London, UK; ${ }^{2}$ Medscape, Medical Education, London, UK; ${ }^{3}$ University College London, UCl Cancer Institute, London, UK

\subsection{6/ijgc-2021-IGCS.254}

Objectives This study determined the knowledge of clinicians on the evidence for, and application of, PARP inhibitors (PARPi) in newly diagnosed ovarian cancer.

Methods A 27-question, online, continuing medical education (CME) self-assessment was developed that included demographic, knowledge, confidence and practice-based multiplechoice questions. Activity was launched for clinicians practising outside of the USA in July 2020 and data collected to October 2020 .

Results 104 oncologists, 46 obstetricians/gynaecologists (obs/ gyn) and 21 other physicians completed the assessment. Participants were divided evenly between academic and community practice, but only $20 \%$ specialized in ovarian cancer. Only $33 \%$ were moderately/very confident (across a 5-point Likert scale) in their ability to select an appropriate PARPi maintenance regimen. Knowledge of key trials was low. 56\% identified the population of the SOLO 1 trial, $37 \%$ patient characteristics, 29\% correct PFS outcome and 43\% most common AEs. $56 \%$ identified the outcome of the PRIMA trial, $33 \%$ patient characteristics, $28 \%$ efficacy in key subpopulations, and 43\% most common G3/4 AEs. 49\% identified the outcome of the PAOLA-1 trial, 44\% efficacy in key subpopulations, and $44 \%$ the $\mathrm{AE}$ profile. Only 32\% identified the appropriate PARPi maintenance regimen for a patient with HRD-ve/BRCA-ve disease following carboplatin/paclitaxel, whilst 77\% identified the appropriate PARPi maintenance strategy following chemotherapy + bevacizumab for BRCA + ve disease. Obs/gyns and other physicians generally performed worse than oncologists in terms of knowledge and confidence.

Conclusions The knowledge and confidence gaps revealed indicate there is a significant need for education to facilitate optimal application of PARPi maintenance strategies in newly diagnosed ovarian cancer. 